\title{
SOBRE DERECHO Y LA AVERIGUACIÓN DE LA VERDAD*
}

\author{
Sebastián Reyes Molina ** \\ Universidad de Uppsala \\ sebastian.reyes@filosofi.uu.se
}

«The secret to keeping authority is to give only orders that you know will be obeyed»
Joe ABERCROMBIE, HaW

RESUMEN. En el ámbito del derecho probatorio la relación entre derecho y verdad ha sido un debate abandonado en los últimos treinta años. Se ha aceptado que existe una conexión entre ambos la cual se traduciría en que el derecho es un medio de averiguación de la verdad de los hechos alegados. La tesis reconstruida en este trabajo ha sido presentada por el profesor Jordi FERRER quien justifica la relación entre derecho y verdad en términos de necesidad para que el derecho pueda motivar la conducta de los ciudadanos. A esta tesis la denomino la teoría de la cláusula estructural de la verdad. En este trabajo se presenta una reconstrucción de esta teoría, dos objeciones a las que se debe enfrentar y cinco posibles interpretaciones de la relación entre derecho y verdad.

Palabras claves: teoría del derecho, proceso legal, verdad, hechos, derecho probatorio.

\section{On Law and the Ascertainment of Truth}

ABSTRACT. In the field of Evidence Law the relation between truth and law has been a somewhat nondebated topic in the past years. It is a given that such connection exists and, it is understood as the notion of the ascertainment of truth of disputed questions of fact through legal evidence. The thesis that I have reconstructed in this paper has been presented by the Prof. Jordi FERRER who grounds the connection between truth and law in the role of the legal system as a tool for guiding the behaviour of the governed. I call this thesis the theory of the structural clause of truth. In this paper I shall present a possible reconstruction of this theory, I shall raise two objections against it and I shall present five possible interpretations of the relation between truth and law.

Keywords: Legal Theory, Truth, Facts, Legal Process, Evidence Law.

* Fecha de recepción: 8 de junio de 2016. Fecha de aceptación: 8 de diciembre de 2016.

** PhD (c) por la Universidad de Uppsala. LL. M., por la Universidad de Génova. LCJS, por la Universidad Austral de Chile. Correo: sebastian.reyes@filosofi.un.se. Quiero agradecer al profesor Álvaro NúÑEZ VAQUERO (Universidad Austral de Chile) y a Donald Bello HutT (King's College London) por sus valiosos comentarios a versiones previas de este trabajo y a la profesora Patricia Mindus (Universidad de Uppsala) y al profesor Jordi FERRER (Universidad de Girona) por sus observaciones y recomendaciones. Una versión previa de este trabajo fue presentado en las I Jornadas de Filosofía del Derecho del Mundo Latino celebrado en Alicante el 26-29 de mayo de 2016. Los errores en los que se ha podido incurrir son de mi responsabilidad. 


\section{COMENTARIOS INTRODUCTORIOS}

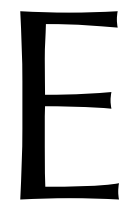

1 presente trabajo tiene por objeto analizar una de las tesis centrales (y hasta cierto punto indiscutidas ${ }^{1}$ del derecho probatorio contemporáneo: la relación necesaria entre derecho y verdad. Esta relación ha sido ampliamente tratada por la literatura especializada la cual entiende que para que el sistema esté orientado a decisiones justas es necesario entonces que las consecuencias jurídicas se apliquen a hechos que han ocurrido en el mundo².

La tesis analizada en este trabajo es la reconstrucción hecha por Jordi FERRER en su libro La valoración racional de la prueba ${ }^{3}$ de la relación existente entre derecho y verdad ${ }^{4}$. FERRER no argumenta a favor de dicha conexión en términos de la justicia de la decisión sino que le atribuye a la verdad un rol central para el derecho en tanto sistema que motiva la conducta de una determinada comunidad. La tesis de FERRER que denominaré como «la tesis de la cláusula estructural de la verdad» (TCEV) posiciona a la búsqueda de la verdad en un sitial preferente con respecto a los demás fines que el derecho promueve ${ }^{5}$. Lo anterior no supone que la búsqueda de la verdad sea el único fin a promover o que este sea absoluto respecto a otros fines. Por el contrario, hay consenso en la literatura especializada de que el derecho promueve diferentes objetivos y que en ocasiones dichos objetivos son contrapuestos ${ }^{6}$. Sin embargo, en «condiciones de normalidad» ${ }^{7}$ la búsqueda de la verdad tendría un lugar preferente sobre el resto de los objetivos protegidos por el derecho.

${ }^{1}$ «Hoy en día nadie minimamente informado puede poner en tela de juicio las ideas maestras de una concepción racionalista del juicio de hecho ni sus implicaciones más evidentes para la práctica jurisdiccional [...] Y todo eso a la luz de dos ideas auténticamente centrales. La primera, que el proceso se orienta a la búsqueda de la verdad, si bien la naturaleza inductiva de los razonamientos probatorios hace que el resultado de la prueba no garantice la certeza absoluta. La segunda, que, aunque el proceso se oriente a la búsqueda de la verdad, al derecho no le interesa solo la averiguación de la verdad, sino también la consecución o protección de otros fines que pueden justificar la introducción de normas sobre la actividad probatoria» (BAYÓN, 2008: 16-7). Las cursivas son mías.

2 Esta postura es defendida en la obra de BENTHAM quien sostenía que el fin del derecho adjetivo (adjective law) es la rectitud de la decisión (Bentham, 1843: 33-34). Por esta se entiende la aplicación de derecho sustantivo (substantive law) a hechos acaecidos en la realidad (ANDERSON, SCHUM y TwINING, 2005: 41) De forma general uno puede encontrar el mismo argumento, quizás mas refinado, en la doctrina contemporánea la que sostiene que la verdad es condición necesaria más no suficiente para alcanzar una decisión justa. A modo de ejemplo vid. TARUFFO, 2010; STEIN, 2005 y Ho, 2008.

3 J. FERRER, La valoración racional de la prueba, Marcial Pons, 2007. Si bien esta tesis ya fue esbozada en trabajos anteriores (FERRER, 2002: 80-1 y FERRER, 2003: 27) es en este libro en el cual la desarrolla de forma más extensa. También es mencionada en FERRER, 2010:3-20.

${ }^{4}$ De forma específica, y expresa, Ferrer sostiene la relación entre prueba y verdad. Sin embargo, como se verá más adelante, su argumento basal es que a través de la prueba jurídica el derecho debe perseguir la verdad si quiere ser útil como herramienta de motivación de la conducta de los gobernados. Es por esto que, en razón de la obra de FERRER, he decidido referirme al binomio derecho-verdad en vez de prueba-verdad. Cabe hacer presente que, en este sentido, la discusión general sobre esta materia ha sido formulada en los términos prueba-verdad.

5 Por sitial preferente me refiero a que la búsqueda de la verdad goza de mayor jerarquía con respecto a la consecución de otras metas promovidas por el derecho.

6 Vid. en este sentido GASCÓN, 2006: 52, FERRER, 2007: 36-40, LAUdAn, 2005: 95, BAYÓN, 2008 y REYes, 2012b.

7 Por condiciones de normalidad me refiero a casos en los cuales las tensiones entre valores opuestos o en competencia no existen o son mínimos. 
Como se explicará mas adelante, FERRER sostiene que la relación entre verdad y derecho es necesaria si este último va a ser un medio útil para determinar la conducta de los sujetos miembros de una comunidad ${ }^{8}$.

El objetivo de este artículo es cuestionar la tesis sostenida en el trabajo de FERRER arguyendo que en los términos en que esta es presentada no es posible sostener la conclusión de que la averiguación de la verdad de los hechos litigiosos es necesaria para que el derecho pueda motivar la conducta. Lo anterior debido a que esta no permite distinguir entre veracidad y verosimilitud en lo que se refiere a la creación del estado psicológico de «sentirse motivado» por el derecho, ni tampoco permite explicar cómo la averiguación de la verdad sortea los problemas del desconocimiento del contenido del derecho y de la indeterminación jurídica. Sin embargo, esto no supone abandonar la noción de relación entre verdad y derecho sino que se pone énfasis en que esta debiese ser objeto de revisión y redefinición para dar cuenta cómo esta noción permea nuestras concepciones sobre la prueba en el derecho. En este espíritu es que se presentan cinco propuestas de redefinición de la relación entre derecho y verdad desde una perspectiva de los usuarios del sistema, a saber: como una norma implícita, como un micro-derecho, como un macro-derecho, como parte de nuestro concepto de derecho y como un ideal regulativo extra-jurídico.

\section{EL MARCO DE LA DISCUSIÓN}

En los últimos treinta años, el derecho continental ha dado un vuelco cuasi-copernicano en el estudio de la prueba jurídica. El llamado «giro epistemológico» (DEI VECCHI, 2013: 235) ${ }^{9}$ experimentado en el estudio de esta disciplina fue de la mano con el cambio de metodología que tradicionalmente se había adscrito al estudio de las reglas que regulan la prueba jurídica ${ }^{10}$. Así, el clásico enfoque del análisis dogmático

${ }^{8}$ La reconstrucción de la relación entre derecho y verdad hecha por FERRER aporta luz desde la teoría del derecho a una de las asunciones preanalíticas quizás más importantes en el derecho probatorio. Su esfuerzo presenta una relación plausible entre dos dimensiones que se han entendido generalmente, tanto en la práctica como en la enseñanza del derecho, como separadas, a saber, lo relativo a la quaestio iuris y la quaestio facti abriendo de esta forma el debate hacia otros ámbitos que generalmente no entran en diálogo.

9 Esto se ve reflejado en un boom en la literatura tanto anglosajona como continental referente a aspectos generales y/o particulares del estudio de la prueba jurídica. Solo a modo de ejemplo vid.: D. SCHUM, A. TERENCE, y W. Twining (2005), Analysis of Evidence, Cambridge: Cambridge University Press; W. TwINING (2006), Rethinking Evidence: Exploratory Essays, Cambridge: Cambridge University Press; L. LAUDAN (2006), Truth, Error and Criminal Law: An Essay on Legal Epistemology, Cambridge: Cambridge University Press; FERRER (2002), Prueba y Verdad en el Derecho, Madrid: Marcial Pons; J. FERRER (2007), La valoración racional de la prueba, Madrid: Marcial Pons; M. TARUFFO (2002), La prueba de los hechos, Madrid: Trota; M. Gascon (1999), Los hechos en el derecho: bases argumentales de la prueba, Madrid: Marcial Pons; D. GonZÁLEZ (2011), Quaestio Facti: Ensayos sobre la prueba, causalidad y acción, México: Fontamara; A. STEIN (2005), Foundations of Evidence Law, Oxford: Oxford University Press; H. L. Ho (2009), A Philosophy of Evidence Law: Justice in the Search of Truth, Oxford: Oxford University Press; C. VÁZQuEz (2015), De la prueba científica a la prueba pericial, Madrid: Marcial Pons; A. KeAne y P. McKeown (2014), The Modern Law of Evidence (10. ${ }^{a}$ ed.), Oxford: Oxford University Press; J. ANDERSon y A. Hopkins (2014), Uniform Evidence Law Guidebook, Oxford: Oxford University Press; S. HAACK (2014), Evidence Matters, Cambridge: Cambridge University Press; B. GARRET (2011), Convicting the Innocent: Where Criminal Procedure Goes Wrong, Boston: Harvard University Press; P. Murphy (2003), Evidence, Proof and Facts, Oxford: Oxford University Press; P. Davis, W. TwinIng y M. VASILAKI (2011), Evidence, Inference and Inquiry, Oxford: Oxford University Press.

${ }_{10}$ Hasta finales del siglo Xx los estudios sobre la prueba en el derecho continental se enmarcaban dentro de la disciplina del derecho procesal. Así, los primeros estudios sobre la prueba fueron llevados a cabo 
de las normas que regulan la prueba jurídica ofrecido por los teóricos del derecho procesal, ha dado paso a una reflexión teórica y crítica con pretensiones normativas sobre qué modelos de admisión y valoración de la prueba son mejores o más aptos para alcanzar determinados fines del sistema, e. g., la verdad de los hechos discutidos, decisiones justas, etc. No es de extrañar entonces que sean los teóricos del derecho los que en la actualidad se han encargado de liderar la discusión en el ámbito del derecho probatorio ${ }^{11}$.

Si bien no existe una escuela o movimiento «oficial» de pensamiento en el derecho probatorio, hay un gran número de académicos que comparten o parecen compartir, explícita o implícitamente, determinados postulados nucleares los cuales permiten trazar líneas en común entre sus trabajos. De forma general, es posible sostener que estos autores se caracterizan por sostener una visión particular de la prueba jurídica: esta debe ser una actividad racional la cual debe ser guiada por reglas epistémicas y criterios de racionalidad general ${ }^{12}$. Los autores que comparten esta pretensión de racionalidad han sido considerados bajo la etiqueta de la tradición racionalista de la prueba $^{13}$.

El paradigma epistemológico adoptado por la generalidad de la tradición racionalista es el cognoscitivista ${ }^{14}$, el cual concibe la actividad probatoria como una actividad eminentemente epistemológica ${ }^{15}$.

El cognoscitivismo, sostiene Gascón, es el

modelo según el cual los procedimientos de fijación de los hechos se dirigen a la formulación de enunciados fácticos que serán verdaderos si los hechos que describen han sucedido y falsos en caso contrario [...] el objetivo que persigue un paradigma cognoscitivista de fijación judicial de los hechos es, pues, la formulación de enunciados fácticos y verdaderos [...] que sean 'fácticos' significa que son una descripción de los hechos acaecidos; es decir, que el juicio de hecho tiene naturaleza descriptiva [...] que sean «verdaderos» significa que los hechos descritos por tales enunciados han tenido lugar (GASCÓN, 1999: $51-53)$.

por profesores especialistas en derecho procesal (siendo el caso italiano quizás el más conocido). Vid. en este sentido Chiovenda, 1923; CARnelutti, 1915; CAlamandrei, 1943, y en Sudamérica, Couture, 1942 y Devis ECHANDÍA, 1974.

11 No solo los teóricos del derecho han demostrado interés en el ámbito probatorio sino que también, entre otros, epistemólogos y psicólogos por medio de la ciencia cognitiva. Los estudios de derecho probatorio contemporáneo han demostrado ser esfuerzos multidisciplinarios e interdisciplinarios.

12 Vid. en este sentido, GASCÓN, 1999 y LAUDAN, 2006. Cabe señalar, siguiendo a TARUFFO, que la elección de una concepción racionalista responde a una elección político-valorativa de un modelo de explicación y estudio de la prueba jurídica, así «cabe subrayar que la adopción de una concepción racionalista —o simplemente racional- de la decisión judiciaria no se puede considerar como una premisa pacífica, que se puede considerar como dada a priori. Al contrario, se trata de una elección evaluativa, de carácter esencialmente ideológico, que tiene profundas implicaciones culturales y políticas» (TARUFFO, 2013: 92).

${ }_{13}$ Quizá los orígenes de esta nomenclatura pueden ser trazados en los trabajos de William TwINING por medio de lo que él ha denominado la «tesis histórica» del derecho probatorio (TwINING, 2006: 75 y ss.) Como sea hoy parece ser una etiqueta aceptada, a veces de forma explícita, por los autores que trabajan estas materias. Esto no quiere decir que exista una «escuela oficial» conocida como «racionalista» en materia de derecho probatorio.

14 Sin embargo, este no es el único modelo. En el seno del debate se han presentado y elaborado concepciones alternativas al modelo cognoscitivista siendo el modelo coherentista el que generalmente se adopta como contrario a la corriente mayoritaria. Vid. (AMAYA, 2013 y 2015).

15 En este sentido, vid. GASCÓN, 2002. 
De acuerdo a esta postura, el procedimiento probatorio, en el contexto de un juicio, desempeña una función cognitiva por cuanto permite al juez conocer o descubrir los hechos a partir de las pruebas rendidas en el proceso por las partes. Es decir,

(e)l término «prueba», en el contexto jurídico, identifica los trámites o actividades que se orientan a acreditar o a determinar (en definitiva, a probar) la existencia o inexistencia de hechos relevantes para adoptar la decisión. Precisamente por eso, frente a la concepción retórica o argumentativa de la prueba [...] desde una perspectiva racional la concepción más adecuada (y no por casualidad la más extendida) es la cognoscitivista, según la cual la prueba se endereza a conocer o acreditar la verdad de (los enunciados sobre) los hechos litigiosos o controvertidos (GASCÓN, 2006: 47).

El modelo cognoscitivista presupone un vínculo entre prueba y verdad. La prueba puede, o al menos debería intentar, alcanzar la verdad de los hechos ${ }^{16}$, es decir, somos capaces de conocer hechos que han ocurrido en el pasado por medio de las pruebas rendidas en un juicio. Existe, entonces, una relación teleológica entre prueba y verdad, de este modo «la finalidad de la prueba como institución jurídica es la de permitir alcanzar el conocimiento acerca de la verdad de los enunciados fácticos del caso» (FERRER, 2006: 33).

Para esto, la tradición racionalista, aboga por un sistema de prueba libre ${ }^{17}$. El derecho no debe regular la actividad probatoria, al menos en lo concerniente a la valoración judicial de la prueba. Esta debe estar constreñida no por normas jurídicas sino por normas de la epistemología general. Así

se deja atrás la imagen subjetivista y puramente potestativa del principio de valoración de la prueba reconociéndose que el descarte de las reglas de tasación legal no implica la exclusión de toda regla, sino más bien la sujeción solo a los criterios de racionalidad de la epistemología general (ACCATINO, 2010: 119).

De este modo la noción de libertad de prueba se refiere a la eliminación de un sistema tasado de la misma. Lo anterior no significa que el juez no esté constreñido por regla alguna sino que las normas jurídicas no deben guiar la valoración de la prueba. Este rol es asignado a otro tipo de reglas: las reglas de la razón y epistemología general ${ }^{18}$.

Por medio de estas reglas la valoración racional de la prueba, apunta a disminuir al máximo la discrecionalidad del juez al momento de determinar los hechos. Es decir, se empeña en expulsar/contener/restringir elementos subjetivos (elementos de la personalidad del juez, criterios políticos, morales, y en general cualquier criterio que escape de las solas reglas epistémicas) que eventualmente puedan influenciar al juzgador al momento de decidir sobre las hipótesis discutidas por considerarlas como obstáculos para alcanzar la verdad.

16 Sin perjuicio de lo anterior, como el juicio se desarrolla en condiciones de incertidumbre, el objetivo de alcanzar la verdad se traduce en la minimización del riesgo de error en las decisiones sobre hechos. Vid., en este sentido, STEIN, 2005: 12.

17 Reconstruido como la «libre valoración de la prueba», esta tesis fue propuesta por BENTHAM en el siglo XIX. Vid. J. BENTHAM, «The Rationale of Evidence», The Works of Jeremy Bentham, vol. 6, London: Simpkin, Marshal \& Co.

18 En líneas generales, un sistema de valoración libre de la prueba viene aparejado con ciertos «límites». Estos son los previstos en el modelo denominado sana crítica: reglas de la lógica, máximas de las experiencias y conocimientos científicamente afianzados. De esta forma el juzgador no podría infringir estos límites al momento de valorar la prueba. 
Para FERRER una concepción racional de la prueba no es el resultado de una preferencia de un determinado modelo ideal de prueba por sobre otro. Al contrario, es lo que el ordenamiento jurídico demanda como garantía del derecho a la prueba:

Sostendré que es el propio sistema jurídico, a través del denominado «derecho a la prueba», el que exige la aplicación de esas reglas de la epistemología o la racionalidad generales [...] para la valoración de la prueba [...]. La idea fundamental es que el ciudadano tiene derecho a mostrar la verdad de los hechos en los que funda su pretensión [cita omitida]. En otras palabras el ciudadano tiene derecho a probar que se han producido, o no, los hechos a los que el derecho vincula consecuencias jurídicas. Solo de este modo puede garantizarse una correcta aplicación del derecho y, [...] una adecuada seguridad jurídica (FERRER, 2007: 53-4) ${ }^{19}$.

Para FERRER la averiguación de la verdad es un criterio de corrección de la decisión judicial. Esto presupone que por medio del proceso judicial es posible averiguar la verdad de los hechos discutidos. El juicio, bajo esta concepción, es una herramienta epistémica que permitiría al juez el conocimiento de hechos pasados, únicos e irrepetibles (REYES, 2012a: 234) ${ }^{20}$.

La TCEV propuesta por FERRER se inscribe dentro de una concepción racionalista de la prueba. De acuerdo a esta si la verdad de los hechos declarados probados es necesaria para validar las decisiones jurídicas entonces se requiere una vía para poder averiguar dicha verdad. Esa vía sería el juicio (o el proceso legal). Solo si se es posible averiguar la verdad de los hechos litigiosos por medio del proceso tiene sentido entender la verdad como un criterio de corrección de la decisión judicial. Ahora bien, que la verdad sea un criterio de corrección de la decisión no dependería, de acuerdo a FERRER, de una preferencia de un modelo sobre otro, sino que responde a una exigencia del sistema jurídico que al consagrar el derecho a la prueba prevería la necesidad de buscar la verdad de los hechos alegados dentro de un juicio.

La reconstrucción presentada permite identificar las bases de una relación entre derecho y verdad. Hasta ahora una de las razones por las cuales la verdad juega un rol, no menor, en el derecho es que permitiría justificar (junto con otras razones) el ejercicio de poder estatal ${ }^{21}$. Sin embargo, la tesis de FERRER pretende explorar las implicancias de la averiguación de la verdad desde el punto de vista de los fines del sistema jurídico.

FERRER identifica siete características definitorias del contexto de la prueba jurídica: 1) El objetivo institucional es la averiguación de la verdad, 2) se determina nor-

19 FERRER no es el único autor que apoya la concepción racional desde un punto de vista del sistema jurídico. Autores como GASCÓN argumentan que la concepción racionalista de la prueba (y el modelo cognoscitivista que pregona) responde a exigencias del sistema jurídico. Para GASCÓN es un criterio de corrección de la decisión judicial de acuerdo a lo dispuesto por el principio de legalidad. Sostiene que «en el modelo epistemológico el fin de la prueba es la averiguación de la verdad. Es cierto que se trata de un fin instrumental, constituye un requisito para que la solución pueda entenderse correcta, al menos en los sistemas regidos por el principio de legalidad. Ello es así porque la solución judicial a los conflictos exigida por el principio de legalidad es la que deriva de la aplicación de una regla jurídica que prevé una determinada consecuencia en presencia de ciertos hechos, de manera que si los hechos a los que se anuda esa consecuencia son falsos, la solución no será correcta: dentro del principio de legalidad» (GASCÓN, 2002: 492). Las cursivas son del autor.

20 Lo anterior no supone que, por medio del proceso, se pueda efectivamente alcanzar la verdad, sino que al menos este debiese estar configurado de tal forma que permita averiguarla.

21 En términos de una «decisión justa», aquí se reconduce a la idea original de BENTHAM sobre rectitude of adjudication. 
malmente la ocurrencia de hechos pasados, 3) el derecho incluye un buen número de reglas jurídicas sobre la prueba, 4) la toma de decisiones sobre la prueba en el proceso está sometida a estricta limitaciones temporales, 5) las partes intervienen en el proceso, 6) la justificación de la decisión adoptada es relativa a un conjunto determinado de elementos de juicio y 7) la decisión que se adopte está dotada de autoridad (FERRER, 2007: 29-40). Debido a que el presente trabajo busca analizar el enfoque que FERRER le da a la relación derecho-verdad, el objeto a estudiar en este trabajo corresponde a la primera característica mencionada en las lineas anteriores: el objetivo institucional es la averiguación de la verdad.

\section{LA TESIS DE LA CLÁUSULA ESTRUCTURAL DE LA VERDAD}

En su reconstrucción, FERRER vincula el fin de la actividad probatoria (alcanzar la verdad de los hechos alegados) con las funciones del derecho en tanto sistema motivador de conductas ${ }^{22}$.

\section{FERRER explica que}

el objetivo institucional del proceso es la averiguación de la verdad. Y ello no puede ser de otra manera, puesto que ese objetivo es estructuralmente necesario para que funcione el propio derecho como mecanismo de motivación de la conducta. Solo si las consecuencias jurídicas previstas por el derecho para acciones determinadas se aplican efectivamente a esas acciones [...] este podrá cumplir su función de mecanismo de resolución de conflictos (FERRER, 2010: 13-4). Las cursivas son mías.

De este modo,

para que ello resulte efectivo, los sistemas jurídicos desarrollados prevén la existencia de órganos específicos - jueces y tribunales - cuya función principal es la determinación de la ocurrencia de esos hechos a los que el derecho vincula consecuencias jurídicas y la imposición de esas consecuencias a los sujetos previsto por el propio derecho [...] solo si el proceso cumple la función de determinar la verdad de las proposiciones referidas a los hechos probados podrá el derecho tener éxito como mecanismo pensado para dirigir la conducta de sus destinatarios. Solo podrá influirse en la conducta de los hombres y mujeres para que no maten si, efectivamente, el proceso cumple la función de averiguar quién mató y le impone la sanción prevista por el derecho.

Por todo ello, la prueba como actividad tendría la función de comprobar la producción de los hechos condicionantes a los que el derecho vincula consecuencias jurídicas o, lo que es lo mismo, determinar el valor de verdad de las proposiciones que describen la ocurrencia de esos hechos condicionantes» (FERRER, 2007: 29-30). Las cursivas son mías.

FERRER reconstruye la relación entre derecho y verdad como necesaria y teleológi$\mathrm{ca}^{23}$. La prueba es un medio para alcanzar la verdad (relación teleológica entre derecho

22 FERRER señala que «seguramente no se discutirá que una de las funciones principales del derecho es dirigir la conducta de los ciudadanos. Se da por supuesto que lo que pretende el legislador al dictar normas jurídicas prescriptivas es que sus destinatarios realicen o se abstengan a realizar ciertas conductas» (FERRER, 2007: 29).

${ }_{23}$ La tesis teleológica supone el descarte de la relación conceptual entre derecho y verdad bajo la cual no es posible sostener que una proposición fáctica ha sido probada si esta no es verdadera. En líneas generales, la diferencia entre las dos tesis recae en que la última — relación conceptual — supone la vinculación entre la verdad y la prueba como resultado (i. e., solo se tendrá una prueba si lo que se declara probado es también verdad). En cambio, la primera plantea una relación entre la verdad y la prueba como actividad probatoria. Si 
y verdad) porque solo si es posible averiguar la verdad de los hechos alegados se podrá motivar la conducta en los términos dictados por las normas que integran un sistema jurídico (relación necesaria entre derecho y verdad).

A esta tesis la denomino la tesis de la cláusula estructural de la verdad (TCEV).

La ventaja del enfoque presentado por FERRER es que la averiguación de la verdad responde a una demanda del sistema jurídico y no a preferencias morales o ideológicas de las ventajas de un sistema por sobre otro. La TCEV ubica a la averiguación no tan solo en el centro de la actividad probatoria, posicionándola como un fin preferente frente a otros posibles fines, sino que, además, como condición necesaria para que el derecho sea efectivo en motivar la conducta de los gobernados.

Así

(s)ólo si las consecuencias jurídicas previstas por el derecho para acciones determinadas se aplican efectivamente a esas acciones (idealmente siempre a esas acciones y nunca a otras), los ciudadanos tendrán motivos para actuar conforme a lo prescrito por el derecho y este podrá cumplir su función de mecanismo de resolución de conflictos. Esta faceta estructural no es compartida por otros valores con los que la averiguación de la verdad puede entrar en conflicto (FERRER, 2007: 82). Las cursivas son mías.

FERRER es consciente que el derecho promueve distintos fines y que estos fines pueden colisionar entre sí,

(a)hora bien, si parece claro ya que la averiguación de la verdad es el objetivo fundamental de la actividad probatoria, también lo es que ese no es el único objetivo [...] decir que algo es la finalidad de una institución o actividad, no excluye que existan otras finalidades o propósitos. Y este es el caso de la regulación jurídica de la prueba, que en muchos casos puede ser entendida como la imposición de excepciones a las reglas de la epistemología general en aras de la protección de otros valores, que comparten protección jurídica con la averiguación de la verdad (FERRER, 2007: 31). Se han omitido las citas.

Sin embargo, la relación necesaria que existiría entre verdad y derecho posiciona a la primera en un sitial preferente con respecto a estos otros valores. Esta posición favorable se traduce en que no sería posible prescindir de la verdad si se quiere tener un sistema jurídico que funcione,

(u)n sistema jurídico puede funcionar perfectamente sin asumir el valor de la inviolabilidad del domicilio o las comunicaciones, por ejemplo. Sería un sistema jurídico indeseable por otros motivos, pero no hay nada que impida que un sistema así sea perfectamente efectivo y eficiente en el cumplimiento de su función motivadora de la conducta. En cambio, el sistema no puede prescindir de la averiguación de la verdad como objetivo institucional del proceso (y, por tanto de la aplicación del derecho) puesto que de otro modo el sistema colapsaría (FERRER, 2007: 82-83). Las cursivas son mías.

En resumen la TCEV sostiene que la relación entre derecho y verdad (averiguación de la verdad) es necesaria para que el derecho sea efectivo como sistema que dirige la conducta de los ciudadanos.

\footnotetext{
bien las dos concepciones coinciden en que para que una proposición se considere probada es necesario que existan elementos de juicio a favor de la misma, divergen en que la teoría de la relación conceptual le agrega un segundo requisito, que la proposición sea verdadera. Como la tesis teleológica no requiere de la verdad de la proposición para ser considerada como probada, perfectamente es posible que una hipótesis sobre los hechos pueda resultar probada, aun cuando sea falsa (FERRER, 2006: 30).
} 
Si bien la TCEV no es el argumento central del libro la valoración racional de la prueba sí es un presupuesto necesario para poder sostener el modelo racionalista que FERRER describe en su obra. Solo en la medida en que la verdad y el derecho estén conectados tiene sentido presentar un modelo racional de valoración de la prueba. Así, la TCEV es la espina dorsal en la cual la obra de FERRER se soporta. Lo cierto es que FERRER no ha desarrollado a fondo esta tesis en su obra y el hecho de que sea generalmente aceptada (o al menos no discutida) en el debate académico ${ }^{24}$ sobre materias de prueba no ayuda a la revisión de sus postulados e implicancias.

Es por esto que este trabajo pone bajo examen crítico la TCEV sosteniendo que en los términos en los que FERRER ha desarrollado esta tesis no es posible concluir que la relación entre derecho y verdad sea necesaria para que el derecho sea efectivo como sistema que dirige la conducta de los gobernados.

\section{EL DERECHO COMO HERRAMIENTA PARA MOTIVAR LA CONDUCTA}

El argumento central de este artículo es que la averiguación de la verdad no es necesaria para motivar la conducta de los agentes. En este sentido, la TCEV es superflua si lo que se quiere justificar es la motivación de la conducta por medio del derecho. Lo anterior no supone eliminar la búsqueda de la verdad de la «ecuación» solo que no ostentaría una posición privilegiada frente a los otros valores que el derecho buscaría promover y proteger. En definitiva no tendría un valor estructural.

Para desarrollar este argumento primero paso a presentar dos formas de entender la TCEV. Estas reconstrucciones giran en torno a dos posibles sentidos que la expresión motivar la conducta puede adoptar a la luz de la relación derecho y verdad.

La primera, es que la TCEV es una tesis que lidia con una noción central en la teoría del derecho: la noción de normatividad. Desde este punto de vista, motivar la conducta sería adscribirle al derecho una propiedad a nivel de conceptual: el derecho tiene fuerza obligatoria. De esta forma, solo sería posible sostener que el derecho guía la conducta si tiene capacidad para obligar a los dirigidos con independencia de las preferencias personales de estos ${ }^{25}$.

En virtud de esta supuesta fuerza obligatoria el derecho solo puede motivar la conducta, si y solo si, está orientado a la búsqueda de la verdad de los hechos discutidos en el proceso. En este sentido, la conexión teleológica entre prueba y verdad se justifica en términos de garantía de la fuerza vinculante del derecho para guiar la conducta de los participantes en una comunidad determinada: en orden de garantizar la fuerza vinculante del derecho es que se debe averiguar la verdad de los hechos discutidos en el proceso judicial ${ }^{26}$.

24 En DeI VeCCHI, 2013: 243 se puede ver una aceptación de la TCEV.

25 Para una revisión crítica de esta tesis vid. REYES, 2016.

26 Esta posibilidad de entender el planteamiento de FERRER abre la puerta al debate de que es lo que él entienda, a su vez, por normatividad del derecho. Lo cierto es que en La valoración racional de la prueba, al menos, no se encuentra explicada esta noción debido a que escapa del objetivo del libro. De esta forma 
La segunda forma de entender la TCEV es la siguiente: el derecho es un sistema de normas entendido como una herramienta para motivar la conducta. En este sentido, el derecho, como cualquier otro sistema de normas con pretensión de control o dirección de conductas (como la religión, códigos morales, etc.), necesita que sea posible la averiguación de la verdad de los hechos si quiere tener éxito en lograr su cometido. Luego, si se requiere averiguar la verdad, se debe disponer entonces mecanismos para su búsqueda y alcance. En este sentido por motivar la conducta se entendería un estado psicológico en los gobernados de conformar su comportamiento a lo dispuesto por el derecho.

Es esta segunda alternativa la que creo que FERRER tiene en mente al presentar la relación entre derecho y verdad en los términos de la TCEV y es en la que me enfocaré para sustentar la postura delineada en las primeras lineas de este apartado. De esta forma, la TCEV es presentada como un argumento instrumental en el sentido de que el derecho es una herramienta para un fin. Este fin es la dirección de las conductas de un grupo de personas. La averiguación de la verdad sirve como medio para lograr ese fin. De acuerdo a la TCEV pareciera ser que la averiguación de la verdad genera un incentivo psicológico en los gobernados, quienes estando al tanto de la posibilidad de que el derecho pueda comprobar sus acciones se sentirán motivados a seguir las prescripciones contenidas en las normas. Si se tiene en cuenta esta línea de argumentación, creo que la TCEV puede ser objeto de al menos dos críticas que debilitarían la relación necesaria entre derecho y verdad.

\subsection{Veracidad $\mathrm{v} / \mathrm{s}$ verosimilitud}

El término motivación utilizado por FERRER es ambiguo. Por una parte, puede ser entendido como la razón para hacer o no hacer algo, por otra, como la justificación de una acción. La diferencia entre ambos sentidos recae, entre otras cosas, en el factor temporal con las que estas suponen operar. El primero es previo a la realización o no de una acción, el segundo es posterior a esta. Si bien FERRER no aclara qué sentido adopta cuando utiliza la noción de motivación creo que él presupone la primera. Esto, debido a que solo se podría modificar o guiar la conducta si se es posible influir en el agente previo a la realización o a la abstención de la conducta objeto de regulación. De este modo, FERRER pareciera referirse, aunque no lo señala explícitamente, a una noción psicológica de motivación. Ya OlIVECRONA nos advertía la naturaleza psicológica de este vínculo «(l)os juristas y los filósofos están conscientes del hecho que la fuerza obligatoria del derecho no pertenece al mundo del tiempo y espacio, el mundo natural. $\mathrm{La}$ conclusión más obvia debe ser que la fuerza vinculante del derecho existe solamente en nuestra imaginación» (OlIVECRONA, 1930: 15) ${ }^{27}$.

\footnotetext{
plantear la discusión en estos términos me parece que excede los propósitos tanto del trabajo de FERRER como de este trabajo. Sin embargo, una cuenta sobre derecho y normatividad se puede encontrar en SPAAK, 2003: 469-485.

${ }_{27}$ En principio es posible sostener que el mundo de las instituciones sociales también se encuentra inserto en un contexto tiempo/espacio puesto que es comprobable empíricamente a la existencia de instituciones sociales que afectan nuestra realidad y vida. Sin embargo, lo que es posible encuadrar en este contexto no son las instituciones sociales per se sino que los comportamientos verificables de una determinada comunidad como
} 
HART sostiene que como generalmente los comportamientos regulados por normas jurídicas son deseables en la vida social, estas se encontrarán investidas de una fuerte presión social para que sean cumplidas. En razón de lo anterior es que los sistemas jurídicos contarían con un contingente de autoridades que velarán por el cumplimiento de lo prescrito por el derecho: «la presión social aparece como una cadena, atando a aquellos que tienen una obligación para que así no actúen libremente. El otro lado de la cadena es sostenida por un grupo de autoridades representativas, quienes insisten en la verificación de algún comportamiento o exigen el castigo» (HART, 2012: 87). En otras palabras, el derecho ejerce presión en los miembros de una comunidad. En este sentido, las normas son (entre otras cosas) elementos que componen el estado psicológico de motivación de las personas y que influencian su comportamiento ${ }^{28}$. Este estado, a su vez, responde a «expresiones imperativas las cuales son usadas en orden de inculcar un determinado comportamiento sobre la gente» (OLIVECRONA, 1930: 21).

En los términos de la TCEV la noción de motivación de la conducta pareciera suponer la creación de un estado psicológico en las personas a seguir las normas jurídicas que conforman un determinado ordenamiento ${ }^{29}$. De esta forma, si averiguar la verdad de los hechos litigiosos implica la creación de un estado psicológico en los gobernados, entonces, para influir en su conducta en la forma prescrita por las del derecho, pareciera ser que no se requiere la verdad para que el derecho tenga éxito en guiar el comportamiento. Bastaría, para lograr el fin propuesto, la apariencia de búsqueda de la verdad. De este modo, no sería necesario que las decisiones/veredictos/sentencias contengan enunciados fácticos verdaderos sino que solo se requeriría que el público (los gobernados) crea que son verdaderos o que parezcan serlos. Es decir, esta apariencia de verdad, i. e., una decisión ordenada de tal forma que parezca haber sido dictada como si hubiese sido el producto de la investigación de la verdad de los hechos discutidos, o lo que es lo mismo, que un observador externo como el usuario del sistema y los miembros de la comunidad, se forme la creencia de que el juicio busca averiguar la verdad, serviría para que los gobernados ajusten su conducta a lo prescrito por las normas (Ho, 2008: 57-59) ${ }^{30}$.

respuesta a lo previsto por lo que conocemos como institución social. Es a esto a lo que se refiere OLIVECRONA cuando sostiene que el derecho no es susceptible de ser entendido como un fenómeno físico del cual podamos dar cuenta con nuestros sentidos.

28 HART presenta una distinción entre sentirse obligado y tener una obligación: la primera corresponde a los motivos psicológicos (creencias) por las cuales se realiza una conducta (HART, 2012: 82-3). La segunda, es una afirmación independiente de los motivos o factores subjetivos de quien se le imputa el deber y «generalmente implica la existencia de una regla» (HART, 2012: 85). Sin embargo, no creo que FERRER sostenga que el derecho de becho crea un «sentimiento de obligación» en las personas sino más bien la motivación a la que hace referencia es una noción, si se quiere, más moderada, en la cual el papel del derecho (o las normas jurídicas) cumple un rol fundacional mas no excluyente con respecto a otras consideraciones de orden extra-jurídico que las personas puedan adoptar con el fin de adecuar su conducta.

29 No pareciera ser que cuando FERRER se refiere a la necesidad de alcanzar la verdad para motivar la conducta este describiendo la existencia de una regla y el comportamiento de los sistemas procesales cuando lidian con la prueba. La TCEV en este aspecto tiene un claro componente normativo, es por esto que adopto la versión psicológica en la cual por motivación se entiende la creación de un estado psicológico de conformar el comportamiento a lo dispuestos por las normas de un sistema jurídico.

${ }_{30} \mathrm{Si}$ bien Ho acepta que a mayor apariencia de que el sistema persigue la verdad existirá mayor conformidad de los gobernados con las normas jurídicas, también es cauto en señalar que el requisito que de becho sea alcance la verdad juega un rol en la justicia de la decisión (Ho, 2008: 60). 
Veracidad y verosimilitud colapsan no haciendo posible la distinción ex ante de algo que parece verdad con algo que es verdad ${ }^{31}$. En este sentido, la TCEV no permite explicar por qué una cuenta verosímil de los hechos no sería idónea para motivar a los gobernados. La búsqueda de la verdad no es necesaria puesto que se podría cumplir el mismo fin por medios distintos a esta en la medida que parezca como si fueran el producto de la investigación veraz ${ }^{32}$.

\subsection{Desconocimiento e indeterminación del derecho}

El segundo argumento que se puede esgrimir en contra de la relación necesaria entre derecho y verdad que postula la TCEV puede ser elaborado en dos partes:

Por una parte, si se requiere que los miembros de una comunidad se comporten acorde a lo prescrito por el derecho, estos debiesen, al menos, tener una idea o estar al tanto de que el proceso cumple la función de averiguar la verdad de los hechos descritos, es decir, al menos debiesen conocer, no a de forma acabada pero si a un nivel general, el funcionamiento del derecho, i. e., saber que el derecho puede alcanzar la verdad, y, al menos, por cuales medios se puede lograr este cometido ${ }^{33}$. Sin perjuicio de lo anterior, esto no parece ser una descripción adecuada de la realidad. La generalidad es que los miembros de una comunidad no posean dicho conocimiento y sin embargo, en su mayoría, se comporten en conformidad a las normas jurídicas (o al menos no en un quebrantamiento constante que pueda poner en jaque el ordenamiento social). En este sentido, no es necesario que los gobernados conozcan que el derecho es capaz de averiguar la verdad de los hechos para ajustar su conducta ${ }^{34}$ a lo previsto por este.

Por otra parte, para que el derecho pueda motivar la conducta se requeriría un mínimo de conocimiento sobre el contenido del derecho por parte de los dirigidos. Este conocimiento, de forma general, recaería tanto en los hechos (que el derecho buscaría tanto promover como desalentar) como en las consecuencias jurídicas que la realización o no realización de dichos hechos conllevarían. Solo de esta forma sería posible saber cómo la conducta de los miembros de la comunidad está o no ajustada a lo dispuesto por las normas jurídicas que la rigen. Vale decir, para planear nuestra conducta acorde al derecho debemos estar en la posibilidad de conocer mínimamente qué dice el derecho. Sostener esta postura es problemática si se reconoce que el dere-

31 Sin perjuicio de lo anterior, verdad y veracidad si bien son nociones estrechamente vinculadas no son sinónimas.

32 Por supuesto, esto no obsta a que si en algún momento se «descubriese» que el derecho solo aparenta la averiguación de la verdad, los gobernados dejaran de guiarse por este. Sin embargo, podría sostenerse que aquí ya no existiría un caso de verosimilitud. Los efectos del acto verosímil solo existen mientras la apariencia de verdad se mantenga.

33 Esto pareciera sugerir que los miembros de la comunidad tengan conocimiento de las reglas procesales de sus respectivos sistemas jurídicos. Lo cierto es que es perfectamente posible no tener conocimiento alguno de cómo funciona el derecho de una determinada comunidad y aun así tener una impresión (o pensar) que el derecho persiga la verdad. Esta actitud puede reconducirse a la primera crítica. Si lo que se requiere para que se ajuste la conducta a las normas es la creencia de que se pueden averiguar los hechos (que infrinjan o cumplan normas) entonces el requisito de que el derecho de hecho averigüe la verdad es superfluo al objetivo planteado.

34 Por ajustar la conducta me refiero en líneas generales a la situación en que los gobernados no infringen de forma sistemática y recurrente lo dispuesto por las normas de un sistema jurídico. 
cho es indeterminado (GUASTINI, 2005: 139-144; LeITER, 2007; DAGAN, 2013) ${ }^{35}$. Esto porque las normas derivadas de la interpretación de disposiciones normativas ${ }^{36}$ pueden arrojar resultados contrarios pero que sin embargo son legalmente válidos (NÚÑEZ VAQUERO, 2013: 15) ${ }^{37}$. Vale decir, ante un mismo caso sería posible concluir que un comportamiento está permitido y prohibido a la vez (GUASTINI, 2014: 91-2). Si bien se podría contra argumentar que la indeterminación del derecho apunta a las consecuencias jurídicas y no a los hechos, no es menos cierto que para determinar qué hechos serán utilizados como premisas del razonamiento jurídico se debe tener de antemano un conocimiento de la categoría deóntica (ordenado, permitido o prohibido) prevista por el derecho, sobre esos hechos. En este sentido, aun si aceptamos que 1) el derecho pueda efectivamente alcanzar la verdad de los hechos alegados y 2) esto sea de conocimiento de los gobernados, la tarea de motivar la conducta será difícil en la medida en que los gobernados, aun teniendo esta información, no estarán en una posición de saber si dichos comportamientos están prohibidos o permitidos ${ }^{38}$.

La TCEV no permite dar cuenta del desconocimiento general del funcionamiento del derecho por parte de los gobernados ni tampoco sortear el problema de la indeterminación jurídica y la imposibilidad de conocer de antemano (antes de una decisión judicial) si un determinado comportamiento está o no prohibido por el derecho ${ }^{39}$.

35 Tratar el fenómeno de la indeterminación del derecho excede los propósitos de este trabajo. Para un desarrollo del tema, vid., entre otros, IGLESIAS VILA, 2006: 55-82; LEITER, 1995: 482-495 y KRESS, 1989: 283337. Cabe hacer la observación de que la doctrina ha presentado diversos tipos de indeterminación. Sin embargo, para efectos de este articulo esta se entenderá de forma general como la imposibilidad general de conocer las consecuencias jurídicas de los actos. Lo anterior está íntimamente relacionada con una de las preocupaciones centrales del realismo jurídico estadounidense, la posibilidad de predecir las decisiones de los tribunales. Vid. a modo de ejemplo K. LLEWELlyn, «A Realistic Jurisprudence: The Next Step», Columbia Law Review, vol. XX, núm. 4 (1930): 448. Si bien puede ser tildado de proto-realista, el autor (quizá más reconocido) que impulsó esta inquietud fue O. W. Holmes en su discurso titulado «The Path of Law». Vid. O. W. HoLmes, The Path of Law and the Common Law (New York: Kaplan, 2008). Sin perjuicio de lo anterior, si bien desde una perspectiva general, no sería posible la predicción de las decisiones judiciales no es menos cierto que (y esto ya requiere de un análisis empírico) si un juez o grupo de jueces llegan a una decisión determinada, basado en estímulos similares para casos similares durante un periodo de tiempo determinado, entonces sí sería plausible sostener que al menos podríamos predecir la decisión que se adoptará, con respecto a ese juez o grupo de jueces en particular, en un caso con similares características.

36 Por interpretar entiendo la adscripción de significado a un texto (GUASTINI, 2011a: 133). En este sentido sostengo que la distinción disposición norma es relevante para entender la noción de indeterminación del derecho. Vid. en este sentido GUASTINI, 2011a:133-156.

37 NÚNEEZ VAQUERO adopta la reconstrucción presentada por LEITER de indeterminación jurídica.

38 En este sentido la noción de indeterminación tiene relación con la posibilidad de conocer de antemano (o no) las consecuencias jurídicas de las acciones realizadas en una determinada comunidad (COMANDUCCI, 1998: 101). Estoy consciente de que podría contra-argumentarse que en un escenario $i d e a l$, en el cual no existe controversia sobre la capacidad epistémica del derecho, como el presentado en la última parte de mi argumento, la averiguación de la verdad sería una condición necesaria más no suficiente para motivar la conducta. Sin embargo, no me detendré a desarrollar las posibles objeciones a este contra-argumento.

39 Una opción sería adoptar una postura cognoscitivista de la interpretación jurídica en la cual el ejercicio de interpretación de disposiciones normativas es un ejercicio de descubrimiento del significado único o correcto de esa disposición (DWORKIN, 1986). Si se sigue esta línea de argumentación entonces se concluye que el derecho está determinado y que es posible para el interprete conocer ese significado único aparejado a la disposición normativa. En este sentido se abre una puerta para que los gobernados puedan, de antemano, conocer el derecho y así conformar sus conductas con lo dispuesto en las normas jurídicas. Lo cierto es que esta postura no permite responder los retos planteados por la tesis de la indeterminación sino que niega de plano que la indeterminación del derecho sea posible. A modo de ejemplo, para ver un desarrollo sobre la discusión: ATIENZA, 2009: 13-26 y AARNIO, 1990: 23-38. 


\section{TCEV REVISITADA}

Las dos objeciones planteadas a la TCEV en el apartado anterior presentan un cuadro en el cual la búsqueda de la verdad no es necesaria para el derecho si lo que se quiere es argumentar a favor de la función motivadora de conductas de un sistema jurídico. En este sentido, el ajuste de comportamiento de los gobernados acorde a lo prescrito por el derecho no depende de la capacidad de este de averiguar la verdad de las conductas de los miembros de una comunidad. $\mathrm{O}$ al menos, como ya se señaló, la verdad no sería necesaria para esto. Lo anterior no supone negar una relación entre derecho y verdad sino que argumentar a favor de una re-examinación de sus presupuestos. Sin perjuicio de que redefinir la relación entre derecho y verdad escapa de los propósitos de este trabajo, en este apartado presentaré cinco reconstrucciones posibles de la TCEV, sin tomar partido por ninguna de estas, tomando como noción central la exigencia de averiguar la verdad.

\subsection{Norma implícita}

La exigencia de averiguar la verdad de los hechos alegados estaría contenida en una norma implícita del sistema. Vale decir, una norma que no es resultado de la interpretación de una disposición normativa ni es consecuencia lógica de una disposición normativa expresa (GUASTINI, 2004: 251) ${ }^{40}$. Estas normas, como su nombre lo indica, no están formuladas de forma expresa en el sistema sino que son el fruto de la interpretación creativa ${ }^{41}$ de una o varias normas explícitas por medio de diversos métodos no deductivos (GUASTINI, 2012:33).

Así

son principios no expresos los que carecen de disposición, es decir, los no explícitamente formulados en alguna disposición constitucional o legislativa, sino elaborados o «construidos» por los intérpretes. Se entiende que los intérpretes, cuando formulan un principio no expreso, no se convierten en legisladores, sino que asumen que tal principio está implícito, latente, en el discurso de las fuentes (GUASTINI, 2001: 138) ${ }^{42}$. Citas omitidas.

En este sentido, la averiguación de la verdad sería una norma implícita del sistema derivada de los derechos fundamentales de corte procesal (derecho a la prueba, debido proceso, defensa, etc.). Desde esta perspectiva se puede hablar de la verdad como valor estructural en la medida en que está implícita en el sistema siendo posible extraer una norma que prescriba la averiguación de la verdad de un conjunto de normas expresas, i. e., derechos fundamentales.

40 Para una cuenta de los tipos de normas implícitas vid. GUASTINI, 1995: 259.

41 No me detendré a analizar la distinción entre interpretación e interpretación como creación jurídica, para esto vid. GUASTINI, 2011b: 157-179. Sin embargo, sostengo que las normas implícitas son un ejercicio de creación jurídica.

42 Si bien la cita presentada habla solo de principios, esto no obsta a que distintos tipos de normas puedan encontrarse de forma implícita en un determinado sistema jurídico. 


\subsection{Micro-derecho a la averiguación de la verdad}

Una segunda forma de entender la TCEV es explorando el concepto de micro y macro derechos. Por micro-derechos entiendo situaciones favorables simples: libertades, inmunidades, potestades y pretensiones ${ }^{43}$, mientras que por macro-derecho entiendo situaciones favorables complejas, vale decir, combinaciones de situaciones favorables simples (BARBERIS, 2008: 14).

De acuerdo a esta interpretación, existiría un micro-derecho a averiguar la verdad de los hechos discutidos en juicio el cual corresponde a las partes litigantes y que se ejercería dentro del proceso. Si bien en este trabajo no analizaré cual posición jurídica favorable corresponde al micro derecho de averiguación de la verdad, una intuición posible es que corresponda a una pretensión cuya contraparte, quien carga con el $d e$ ber, sería el estado, i. e., el juez en tanto órgano estatal. En este esquema el juez debe propender a tratar la prueba, tanto en su admisión como en su valoración, de la forma más acorde para averiguar la verdad. Para lograr este objetivo es que la TCEV propone una concepción racionalista de la prueba.

Este micro-derecho se derivaría, siguiendo a FERRER, del macro-derecho a la prueba (y para el caso de que este no este positivado en un ordenamiento jurídico particular, entonces sería posible de derivar del derecho a defensa o debido proceso) el cual estaría formado por una serie de combinaciones de situaciones favorables simples (micro-derechos) los cuáles son, de acuerdo a FERRER, 1) el derecho a utilizar todas las pruebas de que se dispone para demostrar la verdad de los hechos que funda su pretensión (FERRER, 2007: 54), 2) el derecho a que las pruebas sean practicadas en el proceso (FERRER, 2007:55) y 3) el derecho a una valoración racional de las pruebas practicadas (FERRER, 2007: 56). La verdad sería parte estructural del proceso en su calidad de micro derecho derivado de macro-derechos consagrados en textos autoritativos.

\subsection{Macro-derecho a la verdad}

Una tercera interpretación posible corresponde a que existe un macro-derecho a la verdad. Este macro-derecho estaría compuesto por una serie de posiciones favorables simples de distintas naturaleza que se verán traducidas en las diversas acciones que las partes pueden adoptar en el transcurso del proceso. Así, determinar qué pruebas se presentarán y cómo se desarrollará el examen y contra examen de testigos podrían recaer en categorías de libertades, mientras que tener la opción de presentar prueba, de excluir prueba y de que la prueba presentada sea valorada racionalmente pueden ser pretensiones, a su vez estar dispensado de probar determinados hechos puede verse como inmunidades, etc. La interacción de estas posiciones favorables simples en el contexto del juicio configuraría el macro-derecho a la verdad que le asiste a las partes en el proceso. Este macro-derecho, que se traduce en la averiguación de la verdad por

43 Las llamadas categorías o incidentes bohfeldianos (HOHFELD, 1919). Analizar a fondo las nociones de micro y macro derechos junto con la de derechos-razones escapa del alcance de este trabajo. Para esto vid. BARBERIS, 2008. Una cuenta de esta postura y su relación con concepciones pragmatistas de los derechos se puede encontrar en TUZET, 2013: 11-36. 
medio del proceso, permea toda la instancia del litigio e informa el actuar tanto de las partes como del juez. En este sentido la averiguación de la verdad es estructural puesto que el proceso está orientado a buscar la verdad. Es estructural en relación al proceso que a su vez es la concretización de la posibilidad de defender nuestros derechos o intereses ante un juez.

\subsection{Verdad como parte de nuestro concepto de derecho}

Una cuarta forma de entender la TCEV es sostener que la verdad forma parte de nuestro concepto de derecho. Es decir, no sería posible concebir un ordenamiento jurídico que no tome en cuenta, en algún nivel, la verdad de los hechos a los que busca aplicar las consecuencias jurídicas. En otras palabras, no estaríamos dispuestos a calificar como derecho un conjunto de normas en los que la averiguación de la verdad no jugase ningún papel. Pareciera ser que FERRER implícitamente adhiere a esta postura cuando expresa que «el sistema no puede prescindir de la averiguación de la verdad como objetivo institucional del proceso, y, por tanto de la aplicación del derecho, «puesto que de otro modo el sistema colapsaría» (FERRER, 2007: 83) ${ }^{44}$. Esto refuerza, a su vez, la opción de un modelo racionalista de la prueba, si se asume que este modelo es el más apto para alcanzar la verdad, y la tesis de que la verdad ostenta un lugar de preferencias por sobre otros fines que el derecho promueve. Por otro lado, este enfoque colisiona con concepciones y prácticas clásicas del proceso civil las que generalmente no le otorgan un valor relevante a la persecución de la verdad para el fin del proceso civil, i. e., la resolución de conflictos (TARUFFO, 2013: 97 y ss.) ${ }^{45}$. La adopción de esta postura, también, abre el debate a varios tópicos que exceden el ámbito del derecho probatorio e inciden directamente en clásicas discusiones de la teoría del derecho como la validez material de las normas, la relación entre derecho y moral, predicción de las decisiones judiciales, etcétera.

\subsection{Ideal regulativo extra jurídico}

Por último otro significado posible es entender a la verdad como un ideal regulativo. Ahora bien, la expresión ideal regulativo es ambigua puesto que puede referirse a un principio jurídico o a valores extra jurídicos. Es esta segunda acepción a la que me refiero cuando utilizo la expresión ideal regulativo (KANT, 1781, Crítica de la razón pura, A569-B597; POPPER, 1963: 264 y ss.; HAACK, 1978: 134 y ss.). Que la verdad sea un ideal regulativo supone que es un fin deseable de perseguir pero que sin embargo

44 Las cursivas son mías.

45 De hecho en los países que han desarrollado un estándar de prueba civil se han preocupado no de la consecución de la verdad sino de la existencia de narraciones plausibles o más «convincentes» (e. g., estándar de probabilidad prevalente) excepcionalmente se exige un grado de corroboración mayor (e. $g$., estándar de prueba clara y convincente) sin embargo, dado que generalmente estos estándares están formulados en términos probabilísticos, la probabilidad asignada para determinar cuando un hecho ha sido probado es un reflejo de cuán importante en materia civil es la distribución del error. No analizaré la problemática de los estándares de prueba civil, para esto vid. Reyes, 2015; LARroucau, 2012 y PARDO, 2009. 
cabe la posibilidad de que nunca lo alcancemos. Esto no supone el abandono del ideal regulativo sino que tal es posible cumplir de forma gradual con la pretensión contenida en dicho ideal, $i$. e., averiguar o alcanzar la verdad de los hechos a los cuales se le aplicarán consecuencias jurídicas, i. e., poder estatal. En este sentido la verdad es un valor extrajurídico compartido por una comunidad. Es una pretensión o una expectativa de los usuarios del sistema a que las instituciones que los gobiernan actúen en base a hechos que han ocurrido en la realidad y no ejerzan el poder estatal de forma arbitraria. En este sentido la expectativa de verdad refuerza la confianza en el sistema ${ }^{46}$.

\section{COMENTARIOS FINALES}

La TCEV presentada por FERRER ha sido generalmente aceptada, implícita o explícitamente, por los estudiosos del derecho probatorio en el ámbito de habla hispana. Esta aceptación general ha llevado a una falta de discusión y análisis sobre cuál es el rol de la verdad en el proceso y qué entendemos cuando decimos que el derecho demanda averiguar la verdad de los hechos alegados en un juicio. Se da por sentado, consciente o inconscientemente, que existe una relación entre derecho y verdad y, al menos en el discurso contemporáneo, esto significaría la adopción de un determinado modelo de concepción de la prueba jurídica, i. e., concepción racionalista de la prueba. En líneas generales, este modelo es el reflejo de una agenda de política jurídica en el sentido de que busca cambiar el meta-discurso clásico de la prueba jurídica la cual ha sido estudiada tradicionalmente desde el derecho procesal (al menos en la tradición del Civil Law), a uno de reflexión teórica en el cual se proponen las mejores respuestas a los problemas probatorios que persisten en la práctica de hoy. Lo anterior requiere un arduo trabajo intelectual y bases sólidas de las cuales derivar estudios específicos en la amplia gama que el derecho a la pruebe provee. Una de estas bases es cómo interactúa el derecho y la verdad (si es que interactúan de algún modo).

En este trabajo se ha tratado de presentar una reconstrucción de los argumentos centrales de la tesis de la cláusula estructural de la verdad, dos objeciones posibles que plantean problemas a resolver si lo que se quiere sostener es que: 1) existe una relación entre derecho y verdad y 2) que esta es necesaria para el derecho si queremos que este cumpla su función de motivar la conducta. Por último, se presentaron cinco formas de entender esta relación enfocándome en el rol de averiguación de la verdad que, de acuerdo a quienes sostienen la TCEV, existiría.

Las cinco interpretaciones presentadas del rol estructural que ha sido adscrito por FERRER a la verdad dentro del derecho son de naturaleza distinta, e. g., no es lo mismo un principio o norma implícita que un macro derecho, o un micro derecho que un ideal regulativo extrajurídico o un elemento de nuestro concepto de derecho, y quizás a veces incompatible, $e$. g., si es un macro-derecho entonces no es un ideal regulativo en sentido extra-jurídico.

46 Sin embargo, cabe distinguir tener una expectativa de verdad que de hecho el derecho sea capaz de averiguar la verdad. 
En conclusión, el presente ensayo es simplemente una llamada a no abandonar el estudio de concepciones que, por muy persuasivas y deseables que parezcan a primera vista, son la base para entender cómo funciona el derecho.

\section{BIBLIOGRAFÍA}

AARNIO, A., 1990: «La tesis de la única respuesta correcta y el principio regulativo del razonamiento jurídico», Doxa, 8: 23-38.

ACCATINO, D., 2010: «El modelo legal de justificación de los enunciados probatorios en las sentencias penales y su control a través del recurso de nulidad», en D. ACCATINO, Formación y valoración de la prueba en el proceso penal, Santiago: Legal Publishing, 119-143.

AmayA A., 2013: «Coherence, Evidence and Legal Proof», Legal Theory, 19 (1): 1-43.

- 2015: The Tapestry of Reason: An Inquiry into the Nature of Coherence and its Role in Legal Argument, Oxford: Hart.

ANDERSON, J., y HopKINS, A., 2014: Uniform Evidence Law Guidebook, Oxford: Oxford University Press.

Anderson, T.; Schum, D., y TwInING, W., 2005: Analysis of evidence, UK: Cambridge University Press.

AtIENZA, M., 2009: «Sobre la única respuesta correcta», Jurídicas, 6 (2): 13-26.

BARBERIS, M., 2008: Ética para juristas, Madrid: Trotta.

BAYÓN, J. C., 2008: «Epistemología, Moral y prueba de los hechos: Hacia un enfoque no benthamiano», en Analisi e diritto, 15-34.

Bentham, J., 1843: «The Rationale of Evidence», The Works of Jeremy Bentham, vol. 6, Londres: Simpkin, Marshal \& Co.

CALAmANDReI, P., 1943: Istituzioni di diritto processuale civile secondo il nueovo codice, Milan: CEDAM.

CARNELUTTI, F., 1915: La prova civile: parte generale: il concetto giuridico della prova, Roma: Athenaeum.

Chiovenda, G., 1923: Principii di diritto processuale civile, Napoli: N. Jovene.

Comanducci, P., 1998: «Principios jurídicos e indeterminación del derecho», Doxa, 21 (2), 89-104.

Couture, E., 1943: Fundamentos del derecho procesal civil, Montevideo: B de F., Ltda.

Dagan, H., 2013: Reconstructing American Legal Realism E Rethinking Private Law Theory, Oxford: Oxford University Press.

Davis, P.; Twining, W., y Vasilaki, M., 2011: Evidence, Inference and Inquiry, Oxford: Oxford University Press.

Dei VeCCHI, D., 2013: «Tres discusiones acerca de la relación entre prueba y verdad», Discusiones, 13: 233-264.

Devis Echandía, H., 1976: Teoría general de la prueba judicial, Buenos Aires: Editorial Zabalía.

Dworkin, R., 1986: Law's Empire, Boston: Harvard University Press.

FERRER, J., 2002: Prueba y verdad en el derecho, Madrid: Marcial Pons.

- 2003: «Derecho a la prueba y racionalidad en las decisiones judiciales», Jueces para la democracia, 47: 27-34.

- 2006: «La valoración de la prueba: Verdad de los enunciados probatorios y justificación de la decisión», en M. CARBOnELl, J. OROZCO y R., VÁZQuez, Estudios sobre la prueba, México: UNAM: $1-41$. 
- 2007: Valoración racional de la prueba, Madrid: Marcial Pons.

- 2010: «La prueba es libertad, pero no tanto. Una teoría de la prueba cuasi-benthamiana», en D. Accatino, Formación y valoración de la prueba en el proceso penal, Santiago: Legal Publishing, 3-20.

GARRET, B., 2011: Convicting the Innocent: Where Criminal Procedure Goes Wrong, Boston: Harvard University Press.

GASCÓN, M., 1999: Los hechos en el derecho: bases argumentales de la prueba, Madrid: Marcial Pons.

- 2002: «Sobre el modelo cognoscitivista en la prueba judicial. A propósito de las observaciones de Mario Ruiz», Anuario de Filosofía del Derecho, 19: 489-496.

— 2006: «Freedom of Proof? El cuestionable debilitamiento de la regla de exclusión de la prueba ilícita», en M. CARBONELl, J. OROZCO y R. VÁZQUEZ, Estudios sobre la prueba, México: UNAM: 47-88.

GonZÁLEZ, D., 2011: Quaestio Facti: Ensayos sobre la prueba, causalidad y acción, México: Fontamara.

GUASTINI, R., 1995: «Normas supremas», Doxa, 17 (18): 257-270.

- 2001: Estudios de teoría constitucional, México: Fontamara.

— 2004: «Proyecto para la voz “ordenamiento jurídico" de un diccionario», Doxa, 27 (2): 247-282.

- 2005: «A Sceptical View on Legal Interpretation», en P. ComAnDUCCI y R. GuASTini, Analisi e diritto 2005. Ricerche di giurisprudenza analitica, Giappichelli: 139-144.

- 2011a: «Disposición vs norma», en R. Escudero y S. Pozzolo, Disposición vs Norma, Lima: Palestra: $133-156$.

— 2011b: «Introducción a la teoría de la interpretación. Una formula ambigua», en R. ESCUDERO y S. Pozzolo, Disposición vs Norma, Lima: Palestra, 157-179.

- 2012: «Escepticismo ante las reglas replanteado», Discusiones, 11: 27-57.

- 2014: «Realismo jurídico redefinido», en A. NúÑEZ, Modelando ciencia jurídica, Lima: Palestra: 87-114.

HAACK, S., 1978: Philosophy of Logics, Cambridge: Cambridge University Press.

- 2014: Evidence Matters, Cambridge: Cambridge University Press.

HaRT, H. L. A. (1994) 2012: The Concept of Law, 2. ${ }^{a}$ ed., Londres: Clarendon Law Series.

Ho, H. L., 2008: A Philosophy of Evidence Law: Justice in the Search of Truth, UK: Oxford University Press.

Holmes, O. W., 2008: The Path of Law and the Common Law, Nueva York: Kaplan.

Hohfeld, W. N., 1923: Fundamental Legal Conceptions as Applied in Judicial Reasoning: An Other Legal Essays, New Haven: Yale University Press.

Kant, I. (1781) 2007: Critique of Pure Reason, Reino Unido: Penguin.

Keane, A., y Mckeown, P., 2014: The Modern Law of Evidence (10. ${ }^{a}$ ed.), Oxford: Oxford University Press.

KRESS, K., 1989: «Legal Indeterminacy», California Law Review, 77: 283-337.

LAUDAN, L., 2005: «Por qué un estándar de prueba subjetivo y ambiguo no es un estándar», Doxa, 28: 95-113.

- 2006: Truth, Error and Criminal Law: An Essay on Legal Epistemology, Cambridge: Cambridge University Press.

Larroucau, J., 2012: «Hacia un estándar de prueba civil», Revista Chilena de Derecho, 39 (3): 783-808.

LEITER, B., 2007: Naturalizing Jurisprudence; Essays on American Legal Realism and Naturalism in Legal Theory, Oxford: Oxford University Press. 
Llewellyn, K., 1930: «A Realistic Jurisprudence: The Next Step», Columbia Law Review, vol. XX, núm. 4: 431-465.

Murphy, P., 2003: Evidence, Proof and Facts, Oxford: Oxford University Press.

NúÑEZ, A. 2013: «¿Deciden los jueces por razones políticas?», Jurídicas, 9 (2): 13-31.

Olivecrona, K. 1930: Law as Fact, 1. ${ }^{\text {a }}$ ed., UK: Oxford University Press.

PARDO, M., 2009: «Second Order Proof Rules», Florida Law Review, 61, 1083-1113.

Popper, K., 1963: Conjectures and Refutations: The Growth of Scientific Knowledge, Reino Unido: Routledge \& Kegan Paul.

REYES, S., 2012a: «Juicio como herramienta epistemológica: El rol de la verdad en el proceso», Anuario de Filosofía Jurídica y Social, Sociedad Chilena de Filosofía Jurídica y Social, 30: 221-235.

- 2012b: «Presunción de inocencia y estándares de prueba en el proceso penal: Reflexiones sobre el caso chileno», Revista de Derecho Universidad Austral de Chile, 25 (2), 229-247.

- 2015: «Estándares de prueba y Moral Hazard», Nuevo Derecho, 11 (16), 15-35.

- 2016: «Derecho, motivación de la conducta y quaestio facti» en M. A. NEIRA, Derecho constitucional en la encrucijada, Baja California: Universidad Autónoma de Baja California, Status: en prensa.

SpaAK, T., 2003: «Legal Positivism, Law's Normativity, and the Normative Force of Legal Justification», Ratio Juris, 16 (4): 469-485.

SteIn, A., 2005: Foundations of Evidence Law, Oxford: Oxford University Press.

TARufFo, M., 2002: La prueba de los hechos, Madrid: Trota.

- 2010: Simplemente la verdad, Madrid: Marcial Pons.

- 2013: Verdad, prueba y motivación en la decisión sobre los hechos, México: Cuadernos de divulgación de la Justicia Electoral.

Tuzet, G., 2013: «Una concepción pragmatista de los derechos», Isonomía, 39, 11-36.

TwINING, W., 2006: Rethinking Evidence: Exploratory Essays, Cambridge: Cambridge University Press.

VÁzQUEZ, C., 2015: De la prueba científica a la prueba pericial, Madrid: Marcial Pons.

VILA, M. I., 2006: «La fuente de indeterminación del derecho: una aproximación filosófica», Cuadernos de Derecho Público, 28: 55-82. 